\title{
Performance of Reverse-Link Synchronous DS-CDMA System on a Frequency-Selective Multipath Fading Channel with Imperfect Power Control
}

\author{
Seung-Hoon Hwang \\ Standardization \& System Research Group, UMTS System Research Laboratory, LG Electronics, \\ 533 Hogye-dong, Dongan-gu, Anyang-shi, Kyungki-do, Korea \\ Email: shwang@lge.com \\ Duk Kyung Kim \\ Information \& Communication Engineering, Inha University, 253 Yonghyun-dong, Nam-gu, Inchon, Korea \\ Email: kdk@inha.ac.kr
}

Received 28 July 2001 and in revised form 12 March 2002

\begin{abstract}
We analyze the performance for reverse-link synchronous DS-CDMA system in a frequency-selective Rayleigh fading channel with an imperfect power control scheme. The performance degradation due to power control error (PCE), which is approximated by a log-normally distributed random variable, is estimated as a function of the standard deviation of the PCE. In addition, we investigate the impacts of the multipath intensity profile (MIP) shape and the number of resolvable paths on the performance. Finally, the coded bit error performance is evaluated in order to estimate the system capacity. Comparing with the conventional CDMA system, we show an achievable gain of from $59 \%$ to $23 \%$ for reverse-link synchronous transmission technique (RLSTT) in the presence of imperfect power control over asynchronous transmission for BER $=10^{-6}$. As well, the effect of tradeoff between orthogonality and diversity can be seen according to the number of multipaths, and the tendency is kept even in the presence of PCE. We conclude that the capacity can be further improved via the RLSTT, because the DS-CDMA system is very sensitive to power control imperfections.
\end{abstract}

Keywords and phrases: reverse link synchronous transmission technique, frequency-selective multipath fading, imperfect power control.

\section{INTRODUCTION}

Direct-sequence code-division multiple-access (DS-CDMA) has been considered as the most promising multiple-access scheme for the next generation mobile communications, because of its high flexibility in offering various services with variety of rates and its possibility of achieving greater capacity $[1,2]$. The capacity of DS-CDMA system is mainly limited by multiple-access interference (MAI), and thus techniques to reduce the MAI, such as multiuser detection or interference cancellation, are currently of great interest $[3,4,5]$. In particular, techniques for reverse links have attracted much attention, as the capacity of a reverse voice cellular network link is smaller than that of the forward link. One reason for this is that the code orthogonality is not maintained, because in the reverse link the arrival times of signals from mobile stations (MSs) at a cell site (CS) are different, given the random geographical distribution of MSs within the cell sector.

For terrestrial mobile systems, the reverse link synchronous transmission technique (hereafter, we denote it by
RLSTT) has been proposed to reduce the interchannel interferences over a reverse link [6]. In RLSTT, a closed-form timing control based on a new parameter called the timing control bit is introduced. The DS-CDMA system considered uses an orthogonal reverse-link spreading sequence and timing control algorithm that allows the main paths to be synchronized. Analyses for a single-cell system have shown good performance, especially for an exponentially decaying multipath intensity profile with a large decay factor $[6,7]$. However, the previous analyses have assumed perfect power control, that is, all the users' transmissions arrive with the same power at the CS receiver. In a practical mobile radio environment, an adaptive power control (APC) scheme is always essential to compensate for the distance losses, shadowing, and fading effects. Such a scheme attempts to maintain a constant average performance among the users, and reduce the MAI effect. This results in a randomly varying power control error (PCE), which may be caused by the dynamic range of the APC, the spatial user distributions, and the propagation statistics $[8,9,10,11]$. 
Evaluation of the system capacity degradation due to PCE is the main focus of this paper. To investigate the overall effect of imperfect power control, PCE is considered in terms of the standard deviation of the lognormal distribution. We consider the capacity of a reverse-link synchronous DS-CDMA system over frequency selective Rayleigh fading channels in the presence of imperfect power control scheme. Using the results of [6], which are described in more detail in the appendices of this paper, the system performance degradation as a function of the standard deviation of the PCE is evaluated. We also investigate the impact of the multipath intensity profile (MIP) shape and the number of resolvable paths on the performance of RLSTT, because the extent of orthogonality destruction depends on the multipath channel power delay profile shape and number of resolvable paths. To estimate the system capacity, the coded bit error probability is evaluated and compared with conventional asynchronous CDMA.

The remainder of the paper is organized as follows. In Section 2, channel and system model are described. The performance is analytically derived and evaluated, assuming coherent binary phase shift keying (BPSK) data modulation and a Rake combiner using maximal ratio combining (MRC) in Section 3. Numerical results and conclusions are provided in Sections 4 and 5, respectively.

\section{CHANNEL AND SYSTEM MODEL}

\subsection{Transmitted signal representation}

We assume that the narrowband-modulated signal of each user is first spread by a short orthogonal sequence, and then randomized by a pseudonoise (PN) sequence. Assuming $K$ active users $(k=1,2, \ldots, K)$, the $k$ th transmitted signal is given by

$$
S^{(k)}(t)=\sqrt{2 P_{k}} a(t) W^{(k)}(t) b^{(k)}(t) \cos \left[\omega_{c} t+\phi^{(k)}\right],
$$

where $P_{k}$ is the average transmitted power, $\omega_{c}$ is the common carrier frequency, $\phi^{(k)}$ is the phase angle of the $k$ th modulator, which is assumed to be uniformly distributed in $[0,2 \pi)$, and $a(t)$ is a PN randomization sequence, which is common to all the channels in a cell to maintain the CDMA orthogonality and is expressed as

$$
a(t)=\sum_{j=-\infty}^{\infty} a_{j} p_{T_{c}}\left(t-j T_{c}\right), \quad a_{j} \in\{-1,1\} .
$$

The orthogonal channelization sequence, $W^{(k)}(t)$, is given by

$$
W^{(k)}(t)=\sum_{j=-\infty}^{\infty} w_{j}^{(k)} p_{T_{w}}\left(t-j T_{w}\right), \quad w_{j}^{(k)} \in\{-1,1\}
$$

and user $k^{\prime}$ s data waveform, $b^{(k)}(t)$ is expressed as

$$
b^{(k)}(t)=\sum_{j=-\infty}^{\infty} b_{j}^{(k)} p_{T}(t-j T), \quad b_{j}^{(k)} \in\{-1,1\},
$$

where $T_{w}$ is the chip duration in the orthogonal sequence and $p_{T}(t)$ is a rectangular pulse of unit height and duration $T$. The PN chip interval $T_{c}$ is related to the data bit interval $T$ by the processing gain $N=T / T_{c}$. We assume, for simplicity, that $T_{w}$ equals to $T_{c}$. As well, $w_{j}^{(k)}$ represents the sign of the $j$ th chip for the $k$ th user's orthogonal sequence, $a_{j}$ represents the sign of the $j$ th chip for the PN sequence, and $b_{j}^{(k)}$ is the sign of the $j$ th transmitted symbol for the $k$ th user.

\subsection{Channel model}

The low-pass impulse response of the band-pass channel for the $k$ th user may be written as [12]

$$
h_{k}(\tau)=\sum_{l=0}^{L^{(k)}-1} \beta_{l}^{(k)} e^{j \theta_{l}^{(k)}} \delta\left[\tau-\tau_{l}^{(k)}\right]
$$

Each path is characterized by three variables: its strength $\beta_{l}^{(k)}$, its phase shift $\theta_{l}^{(k)}$, and its propagation delay $\tau_{l}^{(k)}$. A tapped delay line model describes the frequency selective channel with the $l$ th multipath delay of the $k$ th user given by $\tau_{l}^{(k)}=\tau_{0}^{(k)}+l T_{c}[13]$. Assuming Rayleigh fading, the received signal strength of the $k$ th user on the $l$ th propagation path, $l=0,1, \ldots, L^{(k)}-1$, has a probability density function (pdf) given by

$$
p\left(\beta_{l}^{(k)}\right)=\frac{2 \beta_{l}^{(k)}}{\Omega_{l}^{(k)}} \exp \left(-\frac{\left(\beta_{l}^{(k)}\right)^{2}}{\Omega_{l}^{(k)}}\right) .
$$

The parameter $\Omega_{l}^{(k)}$ is the second moment of $\beta_{l}^{(k)}$, that is, $\Omega_{l}^{(k)}=E\left[\left(\beta_{l}^{(k)}\right)^{2}\right]$, with $\sum_{l=0}^{\infty} \Omega_{1}=1$, and we assume it to be related to the second moment of the initial path strength $\Omega_{0}^{(k)}$ for the MIP by

$$
\Omega_{l}^{(k)}=\Omega_{0}^{(k)} e^{-l \delta}, \quad \delta \geq 0 .
$$

The parameter $\delta$ reflects the decay rate of the average path strength as a function of path delay. Note that a more realistic profile model may be given by the exponential MIP, in which the main path occupies more than half of the total received signal power $[12,14,15]$.

\subsection{Rake combiner output}

The receiver is a coherent Rake receiver, where the number of taps $L_{r}$ is a variable that is less than or equal to $L^{(k)}$. The tap weights and phases are assumed to be perfect estimates of the channel parameters. The received signal is represented as

$$
\begin{aligned}
r(t)= & n(t)+\sqrt{2 P} \sum_{k=1}^{K} \sqrt{\lambda_{k}} \sum_{l=0}^{L^{(k)}-1} \beta_{l}^{(k)} a\left[t-\tau_{l}^{(k)}\right] W^{(k)}\left[t-\tau_{l}^{(k)}\right] \\
& \cdot b^{(k)}\left[t-\tau_{l}^{(k)}\right] \cos \left[\omega_{c} t+\varphi_{l}^{(k)}\right],
\end{aligned}
$$

where $\lambda_{k}$ corresponds to the PCE of the $k$ th user, which is 
a random variable due to imperfect power control. We consider $\lambda_{k}$ to be log-normally distributed with standard deviation $\sigma_{\lambda_{k}} \mathrm{~dB}$. In other words, $\lambda_{k}=10^{(x / 10)}$ where the variable $x$ follows a normal distribution. As well, $\phi_{l}^{(k)}$ is the phase of the $l$ th path of the carrier of the $k$ th user, and $n(t)$ is the additive white Gaussian noise (AWGN) with a two-sided spectral density $\eta_{0} / 2$. For the user of interest $(k=1)$, the output of the receiver is given by

$$
\begin{gathered}
U=\sum_{n=0}^{L_{r}-1} \int_{n T_{c}}^{T+n T_{c}} r(t) \beta_{n}^{(1)} a\left(t-n T_{c}\right) W^{(1)}\left(t-n T_{w}\right) \\
\cdot \cos \left[\omega_{c} t+\varphi_{n}^{(1)}\right] d t \\
=\sum_{n=0}^{L_{r}-1}\left\{S^{(n)}+I_{\mathrm{MAI}}^{(n)}+I_{\mathrm{SI}}^{(n)}+I_{\mathrm{NI}}^{(n)}\right\},
\end{gathered}
$$

where

$$
\begin{aligned}
S^{(n)} & =\sqrt{\frac{P \lambda_{1}}{2}} b_{0}^{(1)} T\left\{\beta_{n}^{(1)}\right\}^{2}, \\
I_{\text {MAI }}^{(n)} & =\sum_{k=2}^{K} \sum_{l=0}^{L^{(k)}-1} f(k, l),
\end{aligned}
$$

where

$$
\begin{aligned}
& f(k, l)= \begin{cases}\sqrt{\frac{P}{2}} \sqrt{\lambda_{k}} \beta_{n}^{(1)} \beta_{l}^{(k)} \\
\cdot\left\{b_{-1}^{(k)} R W_{k 1}\left[\tau_{n l}^{(k)}\right]+b_{0}^{(k)} R \hat{W}_{k 1}\left[\tau_{n l}^{(k)}\right]\right\} \\
\cdot \cos \left[\varphi_{n l}^{(k)}\right], \quad \text { if } \tau_{l}^{(k)} \geq \tau_{l}^{(1)} ; \\
\sqrt{\frac{P}{2} \sqrt{\lambda_{k}} \beta_{n}^{(1)} \beta_{l}^{(k)}} \\
\cdot\left\{b_{0}^{(k)} R W_{k 1}\left[\tau_{n l}^{(k)}\right]+b_{1}^{(k)} R \hat{W}_{k 1}\left[\tau_{n l}^{(k)}\right]\right\} \\
\cdot \cos \left[\varphi_{n l}^{(k)}\right], \quad \text { if } \tau_{l}^{(k)} \geq \tau_{l}^{(1)},\end{cases} \\
& I_{\mathrm{SI}}^{(n)}=\sum_{\substack{l=0 \\
l \neq n}}^{L^{(1)}-1} g(l),
\end{aligned}
$$

where

$$
\begin{aligned}
& g(l)=\left\{\begin{array}{l}
\sqrt{\frac{P \lambda_{1}}{2}} \beta_{n}^{(1)} \beta_{l}^{(1)}\left\{b_{-1}^{(1)} R W_{11}\left[\tau_{n l}^{(1)}\right]+b_{0}^{(1)} R \hat{W}_{11}\left[\tau_{n l}^{(1)}\right]\right\} \\
\cdot \cos \left[\varphi_{n l}^{(1)}\right], \quad \text { if } n<l, \\
\sqrt{\frac{P \lambda_{1}}{2}} \beta_{n}^{(1)} \beta_{l}^{(1)}\left\{b_{0}^{(1)} R W_{11}\left[\tau_{n l}^{(1)}\right]+b_{l}^{(1)} R \hat{W}_{11}\left[\tau_{n l}^{(1)}\right]\right\}
\end{array}\right. \\
& I_{\mathrm{NI}}^{(n)}=\int_{n T_{c} \quad \cos \left[\varphi_{n l}^{(1)}\right], \quad \text { if } n>l,}^{T+n T_{c}}, \quad \beta_{n}^{(1)} a\left(t-n T_{c}\right) W^{(1)}\left(t-n T_{w}\right) \\
& \cdot \cos \left[\omega_{c} t+\varphi_{n}^{(1)}\right] d t
\end{aligned}
$$

with $b_{0}^{(1)}$ being the information bit to be detected, $b_{-1}^{(1)}$ is the preceding bit, $\tau_{n l}^{(k)}=\tau_{l}^{(k)}-\tau_{n}^{(1)}, \varphi_{n l}^{(k)}=\varphi_{l}^{(k)}-\varphi_{n}^{(1)}$, and $R W$ and $R \hat{W}$ are Walsh-PN continuous partial cross-correlation functions defined by

$$
\begin{aligned}
& R W_{k 1}(\tau)=\int_{0}^{\tau} a(t-\tau) W^{(k)}(t-\tau) a(t) W^{(1)}(t) d t \\
& R \hat{W}_{k 1}(\tau)=\int_{\tau}^{T} a(t-\tau) W^{(k)}(t-\tau) a(t) W^{(1)}(t) d t .
\end{aligned}
$$

From (9), we see that the output of the $n$th branch, $n=0,1, \ldots, L_{r}-1$, consists of four terms. The first term represents the desired signal component to be detected. The second term represents the MAI from the $(K-1)$ other simultaneous users. The third term is the self-interference (SI) for the reference user. Finally, the last term is the noiseinterference (NI) caused by AWGN.

\section{PERFORMANCE ANALYSIS}

Suppose we have $K$ transmitters able to adjust their timing clock of the main paths to be aligned at the CS by the timing control algorithm [6]. Therefore, in our analysis, the evaluation is carried out for the case in which the arrival time of paths is modeled as asynchronous in every branch (i.e., for multipaths) but as synchronous in the first branch (i.e., for main paths) exceptionally. We first estimate the uncoded bit error performance at different system parameter settings. Assuming perfect interleaving, we then evaluate an upper bound on the coded bit error performance of the system using convolutional codes with hard decision Viterbi decoding.

\subsection{Uncoded BER performance}

Using the Gaussian approximation method, the MAI terms of the first branch and the rest of the branches are modeled as Gaussian processes with variances equal to the MAI variances for $n=0$ and for $n \geq 1$, respectively [6]. Using the random chip model for the Walsh-PN sequences [16] and performing some mathematical operations described in more detail in the appendices, we obtain the following results. The variance of MAI for $n=0$, conditioned on $\beta_{n}^{(1)}$, is

$$
\sigma_{\mathrm{MAI}, 0}^{2}=\frac{E_{b} T(2 N-3)}{12 N(N-1)}\left\{\beta_{0}^{(1)}\right\}^{2} \sum_{k=2}^{K} \lambda_{k} \sum_{l=1}^{L^{(k)}-1} \Omega_{l}^{(k)} .
$$

Similarly, the variance of MAI for $n \geq 1$ is

$$
\sigma_{\mathrm{MAI}, n}^{2}=\frac{E_{b} T(N-1)}{6 N^{2}}\left\{\beta_{n}^{(1)}\right\}^{2} \sum_{k=2}^{K} \lambda_{k} \sum_{l=0}^{L^{(k)}-1} \Omega_{l}^{(k)},
$$

where $E_{b}=P T$ is the signal energy per bit. The conditional variance of $\sigma_{\mathrm{SI}, n}^{2}$ is approximated by [13]

$$
\sigma_{\mathrm{SI}, n}^{2} \approx \frac{E_{b} T}{4 N} \lambda_{1}\left\{\beta_{n}^{(1)}\right\}^{2} \sum_{l=1}^{L^{(k)}-1} \Omega_{l}^{(1)} .
$$


The variance of the AWGN term, conditioned on $\beta_{n}^{(1)}$, is given as

$$
\sigma_{\mathrm{NI}, n}^{2}=\frac{T \eta_{0}}{4}\left\{\beta_{n}^{(1)}\right\}^{2}
$$

Therefore, the output of the receiver $U$, conditioned on $\beta_{n}^{(1)}$, is a Gaussian random process with a mean given by

$$
U_{s}=\sqrt{\frac{E_{b} \lambda_{1} T}{2}} \sum_{n=0}^{L_{r}-1}\left\{\beta_{n}^{(1)}\right\}^{2}
$$

and the variance equal to the sum of the variances of all the interference terms. From (12), (13), (14), and (15), we have

$$
\begin{aligned}
& \sigma_{T}^{2}=\sum_{n=0}^{L_{r}-1}\left(\sigma_{\mathrm{MAI}, n}^{2}+\sigma_{\mathrm{SI}, n}^{2}+\sigma_{\mathrm{NI}, n}^{2}\right) \\
& =\sigma_{\mathrm{MAI}, 0}^{2}+\sum_{n=1}^{L_{r}-1} \sigma_{\mathrm{MAI}, n}^{2}+\sum_{n=0}^{L_{r}-1}\left(\sigma_{\mathrm{SI}, n}^{2}+\sigma_{\mathrm{NI}, n}^{2}\right) \\
& =\left(E_{b} T\right)\left\{\left(\frac{(2-(1 /(N-1))) \sum_{k=2}^{K} \lambda_{k} \sum_{l=1}^{L^{(k)}-1} \Omega_{l}^{(k)}}{12 N}\right.\right. \\
& \frac{\left\{\beta_{0}^{(1)}\right\}^{2}}{\left\{\beta_{0}^{(1)}\right\}^{2}+\sum_{n=1}^{L_{r}-1}\left\{\beta_{n}^{(1)}\right\}^{2}} \\
& +\frac{(N-1) \sum_{k=2}^{K} \lambda_{k} \sum_{l=0}^{L^{(k)}-1} \Omega_{l}^{(k)}}{6 N^{2}} \\
& \frac{\sum_{n=1}^{L_{r}-1}\left\{\beta_{n}^{(1)}\right\}^{2}}{\left\{\beta_{0}^{(1)}\right\}^{2}+\sum_{n=1}^{L_{r}-1}\left\{\beta_{n}^{(1)}\right\}^{2}} \\
& \left.\left.+\frac{\lambda_{1} \sum_{l=1}^{L^{(k)}-1} \Omega_{l}^{(1)}}{4 N}+\frac{\eta_{0}}{4 E_{b}}\right) \sum_{n=0}^{L_{r}-1}\left\{\beta_{n}^{(1)}\right\}^{2}\right\} .
\end{aligned}
$$

The variance of the total interference in (17) becomes

$$
\begin{aligned}
& \sigma_{T}^{2}=\left(E_{b} T \Omega_{0}\right)\left\{\frac{(2 N-3)\left[q\left(L^{(k)}, \delta\right)-1\right] \lambda_{I}}{12 N(N-1)}\right. \\
& \cdot \frac{\left\{\beta_{0}^{(1)}\right\}^{2}}{\left\{\beta_{0}^{(1)}\right\}^{2}+\sum_{n=1}^{L_{r}-1}\left\{\beta_{n}^{(1)}\right\}^{2}}+\frac{(N-1) q\left(L^{(k)}, \delta\right) \lambda_{I}}{6 N^{2}} \\
& \cdot \frac{\sum_{n=1}^{L_{r}-1}\left\{\beta_{n}^{(1)}\right\}^{2}}{\left\{\beta_{0}^{(1)}\right\}^{2}+\sum_{n=1}^{L_{r}-1}\left\{\beta_{n}^{(1)}\right\}^{2}} \\
&\left.+\frac{\left[q\left(L^{(k)}, \delta\right)-1\right] \lambda_{1}}{4 N}+\frac{\eta_{0}}{4 E_{b} \Omega_{0}}\right\} \sum_{n=0}^{L_{r}-1}\left\{\beta_{n}^{(1)}\right\}^{2},
\end{aligned}
$$

where $q\left(L^{(k)}, \delta\right)=\sum_{l=0}^{L^{(k)}-1} e^{-l \delta}=\left(1-e^{-L^{(k)} \delta}\right) /\left(1-e^{-\delta}\right)$. Fur- thermore, if we define

$$
S=\frac{1}{\Omega_{0}} \sum_{n=0}^{L_{r}-1}\left\{\beta_{n}^{(1)}\right\}^{2}
$$

then the received signal-to-noise ratio (SNR) at the output of the receiver may be written as $\sigma_{0} S$,

$$
\begin{aligned}
& \sigma_{0} S=\lambda_{1}\left[\frac{(2 N-3)\left[q\left(L^{(k)}, \delta\right)-1\right] \lambda_{I}}{3 N(N-1)}\right. \\
& \cdot \frac{\left\{\beta_{0}^{(1)}\right\}^{2}}{\left\{\beta_{0}^{(1)}\right\}^{2}+\sum_{n=1}^{L_{r}-1}\left\{\beta_{n}^{(1)}\right\}^{2}} \\
&+\frac{2(N-1) q\left(L^{(k)}, \delta\right) \lambda_{I}}{3 N^{2}} \frac{\sum_{n=1}^{L_{r}-1}\left\{\beta_{n}^{(1)}\right\}^{2}}{\left\{\beta_{0}^{(1)}\right\}^{2}+\sum_{n=1}^{L_{r}-1}\left\{\beta_{n}^{(1)}\right\}^{2}} \\
&\left.+\frac{\left[q\left(L^{(k)}, \delta\right)-1\right] \lambda_{1}}{N}+\frac{\eta_{0}}{E_{b} \Omega_{0}}\right]^{-1} \frac{1}{\Omega_{0}} \sum_{n=0}^{L_{r}-1}\left\{\beta_{n}^{(1)}\right\}^{2}
\end{aligned}
$$

The pdf of $Y=\left\{\beta_{0}^{(1)}\right\}^{2}$ is

$$
p_{Y}(y)=\frac{1}{\Omega_{0}} e^{-y / \Omega_{0}}, \quad y \geq 0
$$

and the pdf of $X=\sum_{n=1}^{L_{r}-1}\left\{\beta_{n}^{(1)}\right\}^{2}$ for exponential MIP is

$$
p_{X}(x)=\sum_{k=1}^{L_{r}-1} \frac{\pi_{k}}{\Omega_{k}} e^{-x / \Omega_{k}},
$$

where

$$
\pi_{k}=\prod_{\substack{i=1 \\ i \neq k}}^{L-1} \frac{x_{k}}{x_{k}-x_{i}}=\prod_{\substack{i=1 \\ i \neq k}}^{L-1} \frac{\Omega_{k}}{\Omega_{k}-\Omega_{i}}
$$

In addition to fast signal fluctuations caused by multipath reflections, slow signal fluctuations exist due to obstruction of the signal by hills and buildings. We can express the received signal power as $P_{k}=P \lambda_{k}$, and $\lambda_{k}$ is the PCE, which is a log-normally distributed random variable $[8,9,10,11]$

$$
p\left(\lambda_{1}\right)=\frac{1}{\sqrt{2 \pi} \sigma_{\lambda_{1}} \lambda_{1}} \exp \left[-\frac{\left(\ln \lambda_{1}-m_{\lambda_{1}}\right)^{2}}{2 \sigma_{\lambda_{1}}^{2}}\right]
$$

The CS receives the desired power of the reference user and the joint interference power. Fenton [17] showed that the pdf of $\lambda_{I}=\sum_{k=2}^{K} \lambda_{k}$ for $K-1$ users is approximately log-normal with the following logarithmic mean and logarithmic variance, which is valid for a logarithmic standard deviation $\sigma$ less than $4 \mathrm{~dB}$ :

$$
p\left(\lambda_{I}\right)=\frac{1}{\sqrt{2 \pi} \sigma_{\lambda_{I}} \lambda_{I}} \exp \left[-\frac{\left(\ln \lambda_{I}-m_{\lambda_{I}}\right)^{2}}{2 \sigma_{\lambda_{I}}^{2}}\right],
$$


where

$$
\begin{aligned}
& \sigma_{I}^{2}=\ln \left(\frac{1}{K-1} e^{\sigma_{\lambda}^{2}}+\frac{K-2}{K-1}\right), \\
& m_{I}=\ln (K-1)+m+\frac{\sigma_{\lambda}^{2}}{2}-\frac{1}{2} \ln \left(\frac{K-2}{K-1}+\frac{1}{K-1} e^{\sigma_{\lambda}^{2}}\right) .
\end{aligned}
$$

The average bit error probability is then

$$
\begin{aligned}
P_{e}^{l} & =\iiint \int P_{e \mid X, Y, \lambda_{1}, \lambda_{I}}^{l} p\left(x, y, \lambda_{1}, \lambda_{I}\right) d x d y d \lambda_{1} d \lambda_{I} \\
& =\iiint \int P_{e \mid X, Y, \lambda_{1}, \lambda_{I}}^{l} p(x) p(y) p\left(\lambda_{1}\right) p\left(\lambda_{I}\right) d x d y d \lambda_{1} d \lambda_{I},
\end{aligned}
$$

where

$$
\begin{aligned}
P_{e \mid X, Y, \lambda_{1}, \lambda_{I}}^{l} & =Q\left(\sqrt{2 \sigma_{0} S}\right), \\
Q(x) & =\frac{1}{\sqrt{2 \pi}} \int_{x}^{\infty} \exp \left(-\frac{u^{2}}{2}\right) d u .
\end{aligned}
$$

From (20), (21), and (22),

$$
P_{e}^{l}\left(\lambda_{1}, \lambda_{I}\right)=\iint_{0}^{\infty} Q\left(\sqrt{2 \sigma_{0} S}\right) \cdot \sum_{k=1}^{L-1} \frac{\pi_{k}}{\Omega_{k}} e^{-x / \Omega_{k}} \cdot \frac{1}{\Omega_{0}} e^{-y / \Omega_{0}} d x d y
$$

Using (24) and (29), we find that

$$
P_{e}^{l}\left(\lambda_{I}\right)=\int_{0}^{\infty} P_{e}^{l}\left(\lambda_{1}, \lambda_{I}\right) \frac{1}{\sqrt{2 \pi} \sigma_{\lambda_{1}} \lambda_{1}} \exp \left[-\frac{\left(\ln \lambda_{1}-m_{\lambda_{1}}\right)^{2}}{2 \sigma_{\lambda_{1}}^{2}}\right] d \lambda_{1} .
$$

Assuming that $z_{1}=\left(\ln \lambda_{1}-m_{\lambda_{1}}\right) / \sqrt{2} \sigma_{\lambda_{1}}$, we can rewrite (30) as

$$
p_{e}^{l}\left(\lambda_{I}\right)=\frac{1}{\sqrt{\pi}} \int_{-\infty}^{\infty} p_{e}^{l}\left(\exp \left(\sqrt{2} \sigma_{\lambda_{1}} z_{1}+m_{\lambda_{1}}\right), \lambda_{I}\right) \exp \left[-z_{1}^{2}\right] d z_{1} .
$$

Therefore, the bit error probability is now given by

$$
\begin{aligned}
p_{e}^{l}= & \frac{1}{\sqrt{\pi}} \int_{-\infty}^{\infty} \frac{1}{\sqrt{\pi}} \\
& \cdot \int_{-\infty}^{\infty} p_{e}^{l}\left(\exp \left(\sqrt{2} \sigma_{\lambda_{1}} z_{1}+m_{\lambda_{1}}\right), \exp \left(\sqrt{2} \sigma_{\lambda_{I}} z_{I}+m_{\lambda_{I}}\right)\right) \\
& \cdot \exp \left[-z_{1}^{2}\right] d z_{1} \exp \left[-z_{I}^{2}\right] d z_{I} .
\end{aligned}
$$

Note that (32) can be calculated using the Hermite polynomial approach, which requires only summation and no integration [18].

\subsection{Coded BER performance}

For convolutional codes with hard decision Viterbi decoding, the bit error rate (BER) transfer characteristic can be

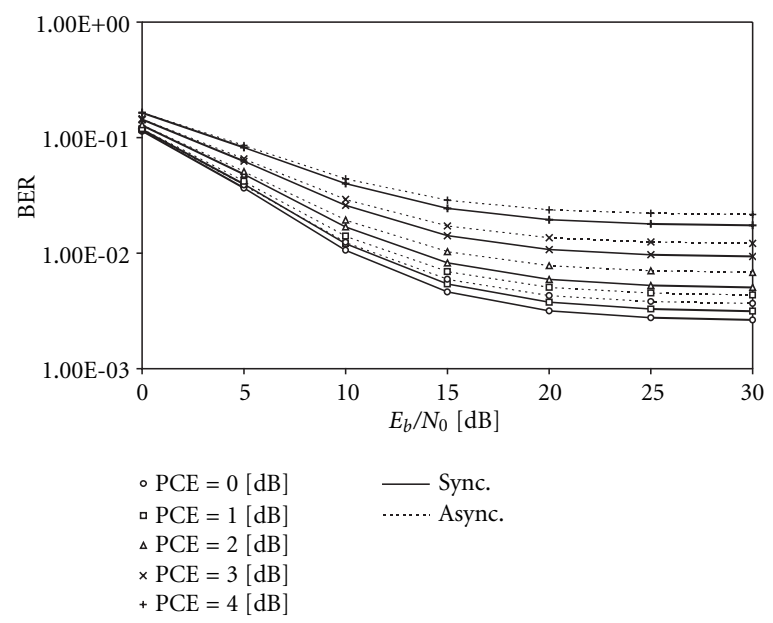

FIgURE 1: Uncoded BER versus $E_{b} / N_{0}$ for different values of PCE standard deviation $(\delta=0.2)$.

upper-bounded by the well-known transfer function bound [12]

$$
p_{0}<\sum_{x=d_{f}}^{\infty} \gamma_{x} P(x)
$$

where $d_{f}$ is the free distance of the code, and $\left\{\gamma_{x}\right\}$ are the coefficients in the expansion of the derivative of $T(D, N)$, the transfer function of the code evaluated at $N=1$ [19]. As well, $P(x)$ is the probability of selecting an incorrect path, which can be bounded by the expression

$$
P(x)<[4 p(1-p)]^{d / 2},
$$

where $p$ is the uncoded BER.

\section{NUMERICAL RESULTS}

In this section, we consider the capacity of a reverselink synchronous DS-CDMA system over frequency selective Rayleigh fading channels in the presence of imperfect power control scheme. The system performance degradation as a function of the standard deviation of the PCE is estimated. We also investigate the effects of the selection of system parameters on the performance of a coherent BPSK Rake receiver with RLSTT in terms of the average BER and the supportable number of users for exponential MIPs. The effect of different values of $\delta$ on the effective total capacity is given. The BER analysis of a conventional asynchronous CDMA system with a diversity technique utilizing maximal ratio combining (MRC) reception can be found in $[12,13]$.

Figures 1 and 2 show the difference in the average uncoded BER performance as a function of $E_{b} / N_{0}$, when $\delta=0.2$ and $\delta=1.0$ are assumed for the different MIPs. Measurements made by Turin et al. [20] in an urban environment indicate that the MIP is exponential. For illustration, we have 


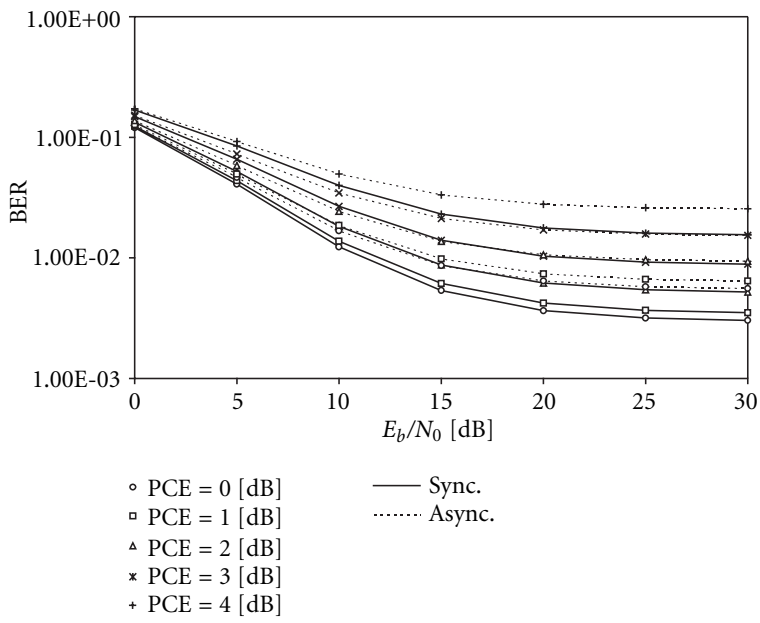

FIgURe 2: Uncoded BER versus $E_{b} / N_{0}$ for different values of PCE standard deviation $(\delta=1.0)$.

chosen the arbitrary parameters $K=24, N=128$, and $L^{(k)}=L_{r}=3$, but we vary the values of $\sigma_{\lambda}$ from 0 to $4 \mathrm{~dB}$. From these figures, we observe that the bit error performance degrades with increased $\sigma_{\lambda}$ values, as expected. In particular, the degradation in performance is marginal when $\sigma_{\lambda}=1 \mathrm{~dB}$. However, for $\sigma_{\lambda}>2 \mathrm{~dB}$, significant degradation is observed. The analytical results derived for asynchronous with the PCE are found to closely match the simulation results obtained by Chockalingam and Milstein in [8]. It is noted that the average BER in RLSTT, when $\sigma_{\lambda}=1 \mathrm{~dB}$, is better than that in the asynchronous transmission when $\sigma_{\lambda}=0 \mathrm{~dB}$ (i.e., perfect power control). It means that the RLSTT with imperfect power control has better performance, compared to non-RLSTT with perfect power control. In addition, as the decay constant $\delta$ of MIP increases, RLSTT in the DS-CDMA reverse link results in a significant BER improvement over the asynchronous transmission even in the presence of imperfect power control. For example, for $E_{b} / N_{0}>15 \mathrm{~dB}$, the average BER of RLSTT when $\sigma_{\lambda}=2 \mathrm{~dB}$ is better than that of the asynchronous transmission when $\sigma_{\lambda}=0 \mathrm{~dB}$.

Figures 3 and 4 show the effect of having a large number of resolvable paths and coherently combining all of them at the Rake receiver, $L^{(k)}\left(=L_{r}\right)$ on the average BER. The average BER is plotted as a function of $L^{(k)}\left(=L_{r}\right)$ for various values of $\sigma_{\lambda}$, when $K=24, N=128, E_{b} / N_{0}=20 \mathrm{~dB}$, and exponential MIP are assumed. The figures illustrate the performance difference between RLSTT and the asynchronous case is smaller as $L^{(k)}\left(=L_{r}\right)$ increases due to the increased MAI resulting from multipath interference. Meanwhile, for large $L^{(k)}\left(=L_{r}\right)$, the potential improvement in performance exists due to the diversity gain from increased frequency selectivity. Therefore, we observe that there are tradeoff between orthogonality and diversity as a function of $L^{(k)}\left(=L_{r}\right)$, and the tendency is kept even in the presence of PCE. However, the

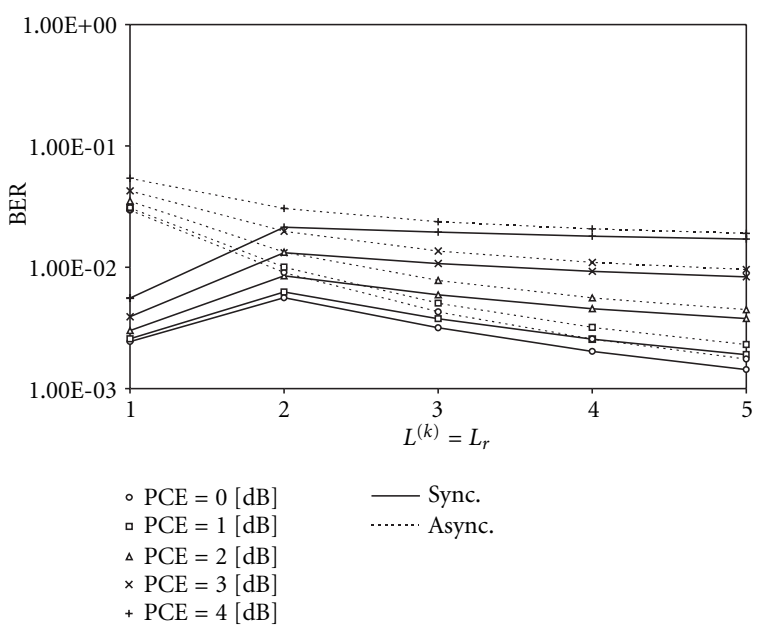

Figure 3: Uncoded BER versus $L^{(k)}=L_{r}$ for different values of PCE standard deviation $(\delta=0.2)$.

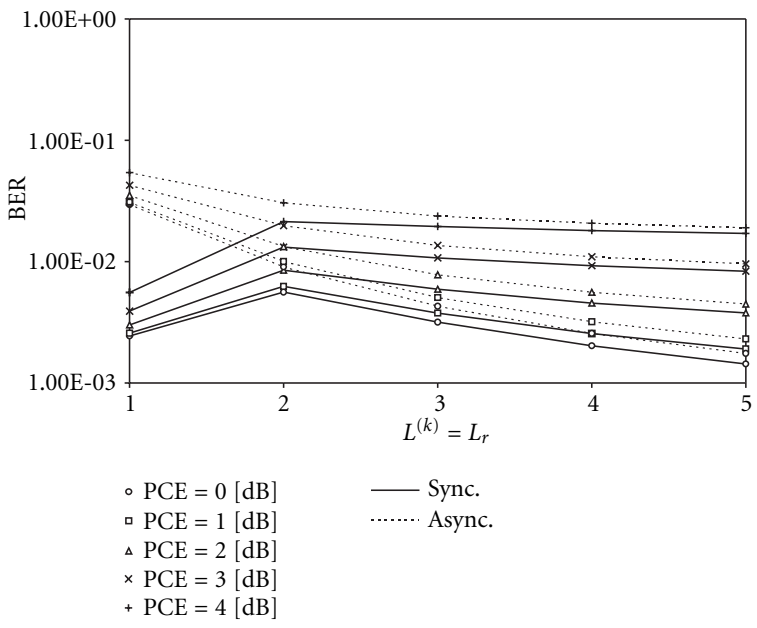

FIGURe 4: Uncoded BER versus $L^{(k)}=L_{r}$ for different values of PCE standard deviation $(\delta=1.0)$.

RLSTT still offers better performance over the asynchronous transmission.

In Figures 5 and 6, the BER is plotted for the situation with perfect and imperfect power control. We see from Figures 5 and 6 that the BER increases with the increase in the imperfection of the power control scheme. This is due to the random nature of the received power in the case of imperfect power control, whereas in the case of perfect power control the received power can be assumed to be deterministic.

We consider the use of a rate $1 / 3$ convolutional code of constraint length 9 on the reverse link [21]. The $\left\{\gamma_{x}\right\}$ coefficients for the corresponding code are taken from [19]. The upper bound on the coded BER performance of the system as 


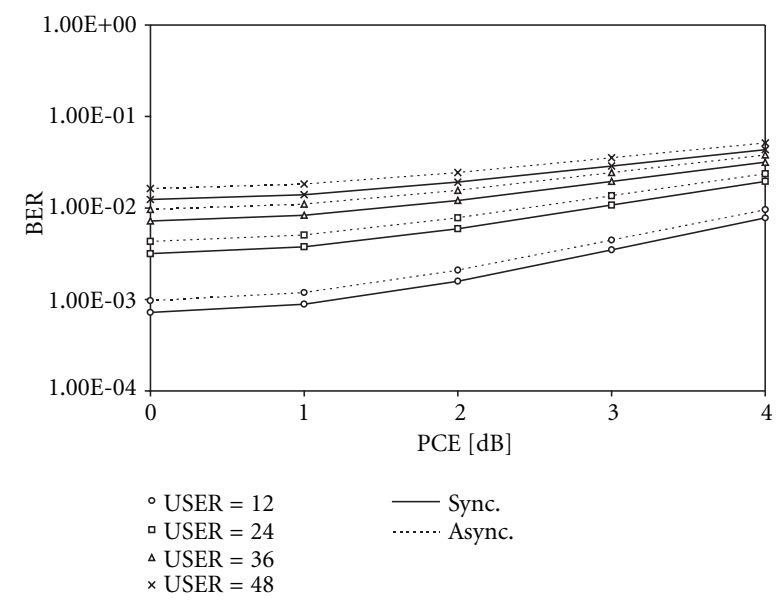

Figure 5: Uncoded BER versus PCE standard deviation for different number of users $(\delta=0.2)$.

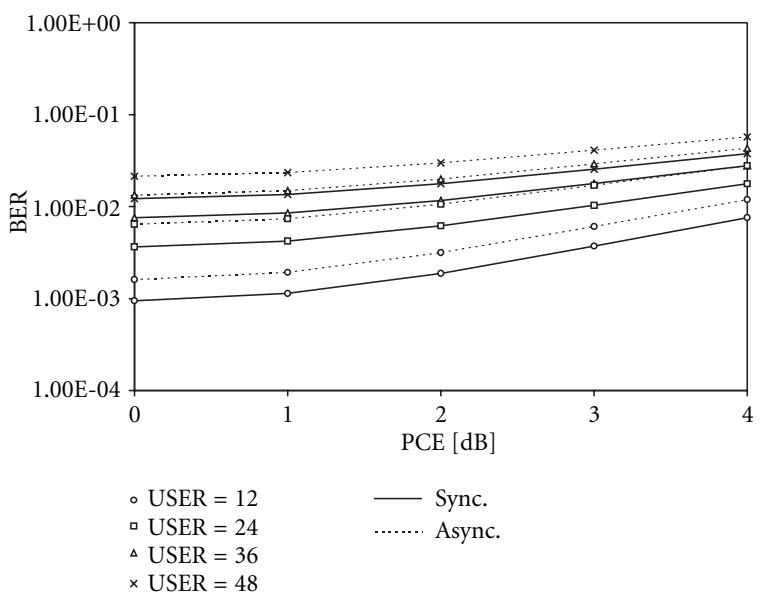

FIGURE 6: Uncoded BER versus PCE standard deviation for different number of users $(\delta=1.0)$.

a function of the number of users, when $E_{b} / N_{0}=20 \mathrm{~dB}$ and $L^{(k)}=L_{r}=3$ can be achieved for different $\sigma_{\lambda}$ values. Based on the results, the system capacity values as a function of $\sigma_{\lambda}$ in $\mathrm{dB}$ for different bit error rates $\left(10^{-3}\right.$ for voice and $10^{-6}$ or $10^{-10}$ for data) are tabulated in Tables 1 and 2 . The system capacity is defined as the number of simultaneous users that can be supported while maintaining an acceptable BER performance needed by the specific application. When RLSTT is employed, a channel with exponential MIP of $\delta=1.0$ has more capacity than a channel with $\delta=0.2$ for the same BER value. For $\mathrm{BER}=10^{-6}$ and $\delta=1.0$, when $\sigma_{\lambda}=0 \mathrm{~dB}$ the capacity improvements are around $50 \%$, while when $\sigma_{\lambda}=2 \mathrm{~dB}$ the improvements increase to $59 \%$. The results have shown that the capacity of DS-CDMA system can be improved by employing RLSTT even in the presence of imperfect power control.
TABLE 1: CDMA system capacity with PCE $\left(\delta=0.2, L^{(k)}=L_{r}=3\right.$, rate $1 / 3$ convolutional code $(K=9)$ with perfect interleaving).

\begin{tabular}{ccccccc}
\hline & \multicolumn{6}{c}{ System capacity } \\
\multicolumn{1}{l}{ BER } & \multicolumn{5}{c}{ Standard derivation of PCE, $\sigma_{\lambda}$} \\
& & $0 \mathrm{~dB}$ & $1 \mathrm{~dB}$ & $2 \mathrm{~dB}$ & $3 \mathrm{~dB}$ & $4 \mathrm{~dB}$ \\
\hline $10^{-3}$ & Sync. & 67 & 64 & 56 & 46 & 36 \\
Voice & Async. & 54 & 51 & 46 & 39 & 30 \\
\hline $10^{-6}$ & Sync. & 39 & 36 & 32 & 25 & 18 \\
Data & Async. & 32 & 30 & 26 & 20 & 15 \\
\hline \multirow{2}{*}{$10^{-10}$} & Sync. & 21 & 20 & 16 & $<12$ & $<12$ \\
& Async. & 17 & 16 & 13 & $<12$ & $<12$ \\
\hline
\end{tabular}

TABLE 2: CDMA system capacity with PCE $\left(\delta=1.0, L^{(k)}=L_{r}=3\right.$, rate $1 / 3$ convolutional code $(K=9)$ with perfect interleaving).

\begin{tabular}{ccccccc}
\hline \multicolumn{1}{c}{ BER } & \multicolumn{5}{c}{ System capacity } \\
\multicolumn{1}{l}{ Standard derivation of PCE, $\sigma_{\lambda}$} \\
& & $0 \mathrm{~dB}$ & $1 \mathrm{~dB}$ & $2 \mathrm{~dB}$ & $3 \mathrm{~dB}$ & $4 \mathrm{~dB}$ \\
\hline $10^{-3}$ & Sync. & 81 & 78 & 69 & 57 & 44 \\
Voice & Async. & 49 & 47 & 42 & 35 & 27 \\
\hline $10^{-6}$ & Sync. & 42 & 40 & 35 & 29 & 20 \\
Data & Async. & 28 & 26 & 22 & 18 & 13 \\
\hline \multirow{2}{*}{$10^{-10}$} & Sync. & 21 & 20 & 17 & 12 & $<12$ \\
& Async. & 15 & 14 & $<12$ & $<12$ & $<12$ \\
\hline
\end{tabular}

\section{CONCLUSIONS}

In this paper, we have considered the effect of imperfect power control in the performance of reverse-link synchronous DS-CDMA system in Rayleigh multipath fading channel. Multiple-access and self-noise interference were modeled as additional Gaussian noise. Under these assumptions, the performance of the coherent system was derived in terms of the uncoded BER and the capacity from the coded BER. The results indicate that in Rayleigh fading with perfect power control, RLSTT shows capacity improvements from $50 \%$ to $22 \%$ for BER $=10^{-6}$. When RLSTT is employed in imperfect power control, it shows more capacity gains from $59 \%$ to $23 \%$. It means that RLSTT with imperfect power control has better performance, compared to non-RLSTT with perfect power control. As well, the effect of tradeoff between orthogonality and diversity can be seen according to the number of multipaths, and the tendency is kept even in the presence of PCE. Finally, we conclude that the capacity can be somewhat further increased via the RLSTT, because the DS-CDMA system is very sensitive to power control imperfections. 


\section{APPENDICES}

\section{A. THE AVERAGE CROSS-CORRELATION PARAMETER}

IN (12)

Let the discrete aperiodic cross-correlation $C W_{k, 1}(i)$ be

$$
C W_{k, 1}(i)= \begin{cases}\sum_{j=0}^{N-1-i} a_{j} w_{j}^{(k)} a_{j+i} w_{j+i}^{(1)}, & 0<i \leq N-1, \\ \sum_{j=0}^{N-1+i} a_{j-i} w_{j-i}^{(k)} a_{j} w_{j}^{(1)}, & -(N-1) \leq i<0, \\ 0, & i=0 \text { or otherwise. }\end{cases}
$$

Let $I_{k}$ be the correlator output resulting from the $k$ th interferer. Then the variance of $I_{k}$ is given by

$$
\begin{gathered}
\operatorname{Var}\left(I_{k}\right)=\frac{P}{2} E\left[\left\{b_{-1}^{(k)} R W_{k 1}\left(\tau_{n l}^{(k)}\right)\right.\right. \\
\left.\left.+b_{0}^{(k)} R \hat{W}_{k 1}\left(\tau_{n l}^{(k)}\right)\right\}^{2}\right] E\left[\cos ^{2}\left(\varphi_{n l}^{(k)}\right)\right] \\
=\frac{P T_{c}^{2}}{12(N-1)}\left[\sum_{i=1}^{N-1} C W_{k, 1}^{2}(1+i-N)\right. \\
+C W_{k, 1}(1+i-N) \\
\cdot C W_{k, 1}(i-N)+C W_{k, 1}^{2}(i-N) \\
+C W_{k, 1}^{2}(i+1)+C W_{k, 1}^{2}(i) \\
\left.+C W_{k, 1}(i) C W_{k, 1}(i+1)\right] .
\end{gathered}
$$

The factor $1 /(N-1)$ and the summation from $i=1$ to $N-1$ in (A.2) arise from the existence of $N-1$ chip intervals in time $\left[T_{c}, T\right]$, and $\tau_{0 l}^{(k)}$ can fall into any one of them with equal probability. Using the random chip model for the Walsh-PN sequences [17], making $\operatorname{Var}\left(I_{k}\right)$ a random variable, and taking the expectation of (A.2)

$$
\begin{aligned}
E\left[\operatorname{Var}\left(I_{k}\right)\right]= & \frac{P T^{2}}{12 N^{2}(N-1)} \\
\cdot E\left[\sum_{i=1}^{N-1} C W_{k, 1}^{2}(1+i-N)+C W_{k, 1}(1+i-N)\right. & \quad \cdot C W_{k, 1}(i-N)+C W_{k, 1}^{2}(i-N) \\
& \quad+C W_{k, 1}^{2}(i+1)+C W_{k, 1}^{2}(i) \\
& \left.\quad+C W_{k, 1}(i) C W_{k, 1}(i+1)\right] \\
= & \frac{P T^{2}}{12 N^{2}(N-1)} \\
& \cdot\left[3 \times \frac{N(N-1)}{2}+\frac{(N-2)(N-1)}{2}-1\right] \\
= & \frac{P T^{2}\left(2 N^{2}-3 N\right)}{12 N^{2}(N-1)}=\frac{P T^{2}}{12 N^{3}} \frac{(2 N-3) N^{2}}{(N-1)}
\end{aligned}
$$

where $C W_{k, 1}(0)=0$,

$$
\begin{aligned}
\sum_{i=1}^{N-2} E\left(C W_{k, 1}^{2}(1+i-N)\right) & =\sum_{i=1}^{N-2} \sum_{m=0}^{i} E(1) \\
& =\sum_{i=1}^{N-2}(i+1)=\frac{(N-1) N}{2}-1, \\
\sum_{i=1}^{N-1} E\left(C W_{k, 1}^{2}(i)\right) & =\sum_{i=1}^{N-1} \sum_{m=0}^{N-1-i} E(1) \\
& =\sum_{i=1}^{N-1}(N-i)=\frac{(N-1) N}{2}, \\
\sum_{i=1}^{N-1} E\left(C W_{k, 1}^{2}(i-N)\right) & =\sum_{i=1}^{N-1} \sum_{m=0}^{i-1} E(1) \\
& =\sum_{i=1}^{N-1} i=\frac{(N-1) N}{2}, \\
\sum_{i=1}^{N-1} E\left(C W_{k, 1}^{2}(i+1)\right) & =\sum_{i=1}^{N-2} \sum_{m=0}^{N-i-2} E(1)=\sum_{i=1}^{N-2}(N-i-1) \\
& =\frac{(N-2)(N-1)}{2} \\
\sum_{i=1}^{N-2} E\left(C W_{k, 1}(1+i-N)\right. & \left.C W_{k, 1}(i-N)\right) \\
& =\sum_{i=1}^{N-1} E\left(C W_{k, 1}(i) C W_{k, 1}(i+1)\right)=0 .
\end{aligned}
$$

Thus, we find that

$$
r_{k 1}(N) \approx \frac{2 N-3}{N-1} N^{2}=\left(2-\frac{1}{N-1}\right) N^{2} .
$$

\section{B. THE AVERAGE CROSS-CORRELATION PARAMETER IN (13)}

Similarly, we can show that

$$
\begin{gathered}
r_{k 1}(N)=2 N(N-1), \\
\operatorname{Var}\left(I_{k}\right)=\frac{P}{2} E\left[\left\{b_{-1}^{(k)} R W_{k 1}\left(\tau_{n l}^{(k)}\right)\right.\right. \\
\left.\left.+b_{0}^{(k)} R \hat{W}_{k 1}\left(\tau_{n l}^{(k)}\right)\right\}^{2}\right] E\left[\cos ^{2}\left(\varphi_{n l}^{(k)}\right)\right] \\
=\frac{P T^{2}}{12 N^{3}}\left[\sum_{i=0}^{N-1} C W_{k, 1}^{2}(1+i-N)\right. \\
+C W_{k, 1}(1+i-N) C W_{k, 1}(i-N) \\
+C W_{k, 1}^{2}(i-N)+C W_{k, 1}^{2}(i+1) \\
\left.+C W_{k, 1}^{2}(i)+C W_{k, 1}(i) C W_{k, 1}(i+1)\right] .
\end{gathered}
$$

The factor $1 / N$ and the summation from $i=0$ to $N-1$ in 
(B.2) are required because there are $N$ chip intervals in time $[0, T]$, and $\tau_{n l}^{(k)}$ can fall into any one of them with equal probability

$$
\begin{aligned}
E\left[\operatorname{Var}\left(I_{k}\right)\right]= & \frac{P T^{2}}{12 N^{3}} \\
& \cdot E\left[\sum _ { i = 0 } ^ { N - 2 } \left\{C W_{k, 1}^{2}(1+i-N)\right.\right. \\
& \left.+C W_{k, 1}(1+i-N) C W_{k, 1}(i-N)\right\} \\
& +\sum_{i=0}^{N-1}\left\{C W_{k, 1}^{2}(i-N)+C W_{k, 1}^{2}(i+1)\right\} \\
& \left.+\sum_{i=1}^{N-1}\left\{C W_{k, 1}^{2}(i)+C W_{k, 1}(i) C W_{k, 1}(i+1)\right\}\right] \\
= & \frac{P T^{2}}{12 N^{3}}\left[4 \times \frac{N(N-1)}{2}\right]=\frac{P T^{2}}{6 N^{2}}(N-1) \\
= & \frac{P T^{2}}{12 N^{3}} 2 N(N-1),
\end{aligned}
$$

where $C W_{k, 1}(0)=0$,

$$
\begin{aligned}
\sum_{i=0}^{N-2} E\left(C W_{k, 1}^{2}(1+i-N)\right) & =\sum_{i=0}^{N-2} \sum_{m=0}^{i} E(1) \\
& =\sum_{i=0}^{N-2}(i+1)=\frac{(N-1) N}{2}, \\
\sum_{i=1}^{N-1} E\left(C W_{k, 1}^{2}(i)\right) & =\sum_{i=1}^{N-1} \sum_{m=0}^{N-1-i} E(1) \\
& =\sum_{i=1}^{N-1}(N-i)=\frac{(N-1) N}{2}, \\
\sum_{i=0}^{N-1} E\left(C W_{k, 1}^{2}(i-N)\right) & =\sum_{i=0}^{N-1} E\left(C W_{k, 1}^{2}(i+1)\right) \\
& =\frac{(N-1) N}{2}, \\
\sum_{i=0}^{N-2} E\left(C W_{k, 1}(1+i-N)\right. & \left.C W_{k, 1}(i-N)\right) \\
& =\sum_{i=1}^{N-1} E\left(C W_{k, 1}(i) C W_{k, 1}(i+1)\right) .
\end{aligned}
$$

\section{REFERENCES}

[1] E. Dahlman, P. Beming, J. Knutsson, F. Ovesjo, M. Persson, and C. Roobol, "WCDMA-the radio interface for future mobile multimedia communications," IEEE Trans. Vehicular Technology, vol. 47, no. 4, pp. 1105-1118, 1998.

[2] F. Adachi, K. Ohno, A. Higashi, T. Dohi, and Y. Okumura, "Coherent multicode DS-CDMA mobile radio access," IEICE Trans. Communications, vol. E-79-B, pp. 1316-1325, September 1996.
[3] R. A. Iltis, "Performance of constrained and unconstrained adaptive multiuser detectors for quasi-synchronous CDMA," IEEE Trans. Communications, vol. 46, no. 1, pp. 135-143, 1998.

[4] H. Sari, F. Vanhaverbeke, and M. Moeneclaey, "Extending the capacity of multiple access channels," IEEE Communication Magazine, vol. 38, pp. 74-82, January 2000.

[5] H. Sari, F. Vanhaverbeke, and M. Moeneclaey, "Multiple access using two sets of orthogonal signal waveforms," IEEE Communications Letters, vol. 4, no. 1, pp. 4-6, 2000.

[6] E. K. Hong, S. H. Hwang, K. J. Kim, and K. C. Whang, "Synchronous transmission technique for the reverse link in DSCDMA terrestrial mobile systems," IEEE Trans. Communications, vol. 47, no. 11, pp. 1632-1635, 1999.

[7] D. K. Kim, S. H. Hwang, E. K. Hong, and S. Y. Lee, "Capacity estimation of uplink synchronized CDMA system with fast TPC and two antenna diversity reception," IEICE Trans. Communications, vol. E84-B, no. 8, pp. 2309-2312, 2001.

[8] A. Chockalingam and L. B. Milstein, "Capacity of DS-CDMA networks on frequency selective fading channels with openloop power control," in Proc. IEEE Int. Conf. Communications, vol. 2, pp. 703-707, Seattle, Wash, USA, June 1995.

[9] N. Kong and L. B. Milstein, "Error probability of multicell CDMA over frequency selective fading channel with power control error," IEEE Trans. Communications, vol. 47, pp. 608617, April 1999.

[10] A. Abrardo and D. Sennati, "On the analytical evaluation of closed loop power control error statistics in DS-CDMA cellular systems," IEEE Trans. Vehicular Technology, vol. 49, no. 6, pp. 2071-2080, 2000.

[11] A. Chockalingam, P. Dietrich, and R. R. Milstein, L. B. Rao, "Performance of closed loop power control in DS-CDMA cellular systems," IEEE Trans. Vehicular Technology, vol. 47, no. 3, pp. 774-789, 1998.

[12] J. G. Proakis, Digital Communications, McGraw-Hill, New York, NY, USA, 1983.

[13] T. Eng and L. B. Milstein, "Coherent DS-CDMA performance in Nakagami multipath fading," IEEE Trans. Communications, vol. 44, no. 9, pp. 1117-1129, 1996.

[14] D. Parsons, The Mobile Radio Propagation Channel, AddisonWesley, New York, NY, USA, 1992.

[15] Rec. ITU-R M. 1225, "Guideline for evaluation of radio transmission technologies for IMT-2000," 1997.

[16] M. B. Pursley, "Performance evaluation for phase-coded spread-spectrum multiple-access communication-Part I: System analysis," IEEE Trans. Communications, vol. 25, no. 8, pp. 795-799, 1977.

[17] R. Prasad, CDMA for Wireless Personal Communications, Artech House Publishers, Boston-London, 1996.

[18] M. Abramowitz and I. A. Stegun, Handbook of Mathematical Functions, National bureau of standards applied mathematics series. Dover, New York, NY, USA, 1965.

[19] J. Conan, "The weight spectra of some short low rate convolutional codes," IEEE Trans. Communications, vol. 32, no. 9, pp. 1050-1053, 1984.

[20] G. Turin, F. Clapp, T. Johnston, S. Fine, and D. Lavry, "A statistical model of urban multipath propagation," IEEE Trans. Vehicular Technology, vol. 21, no. 1, pp. 1-9, 1972.

[21] R. Padovani, "Reverse link performance of IS-95 based cellular system," IEEE Personal Communications, vol. 1, no. 3, pp. 28-34, 1994, Third quarter. 
Seung-Hoon Hwang was born in Seoul, Korea on February 26, 1969. He received his B.S., M.S., and Ph.D. degrees in electrical engineering from Yonsei University in 1992, 1994, and 1999, respectively. His Ph.D. thesis is entitled Performance Evaluation of a Synchronous DS-CDMA System in a Mobile Radio Channel. Since 1999, he has worked for LG Electronics where he is now a Research Engineer in UMTS System Labora-

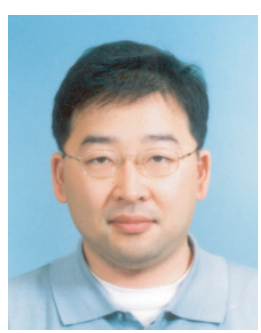
tory, LG R\&D Center, participating in IMT-2000 physical layer standardization activities. His research interests include interference cancellation techniques for DS-CDMA and various aspects of wideband/broadband CDMA.

Duk Kyung Kim received his B.S. degree in electrical engineering from Yonsei University, Seoul, Korea, in 1992, and the M.S. and Ph.D. degrees from the Korea Advanced Institute of Science and Technology (KAIST), in 1994 and 1999, respectively. From 1999 to 2000, he was a Postdoctoral Researcher at the Wireless Laboratories, NTT DoCoMo, Japan. From 2000 to 2002, he worked at

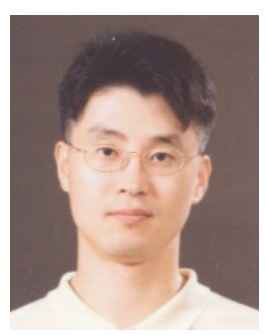
R\&D center, SK Telecom, Korea and in-

volved in the standardization in 3GPP and also in $4 \mathrm{G}$ system development in SK Telecom. In 2002, he joined in the faculty of Inha University. He was interested in asynchronous transfer mode (ATM) network and ATM-based personal communication service (PCS) network. His research interests now include system performance evaluation at link/system level, handoff modeling/management, power control, and multimedia provision in the next generation wireless systems. 